\title{
Lipid environment induces ER stress, TXNIP expression and inflammation in immune cells of individuals with type 2 diabetes
}

\author{
Anaïs Szpigel ${ }^{1,2} \cdot$ Isabelle Hainault $^{1} \cdot$ Aurélie Carlier $^{3} \cdot$ Nicolas Venteclef $^{1}$. \\ Anne-Françoise Batto ${ }^{1}$ - Eric Hajduch ${ }^{1}$. Catherine Bernard ${ }^{2}$ - Alain Ktorza ${ }^{2}$. \\ Jean-François Gautier $^{1,4}$. Pascal Ferré ${ }^{1,5}$. Olivier Bourron ${ }^{1,3} \cdot$ Fabienne Foufelle $^{1}$
}

Received: 20 July 2017 / Accepted: 24 August 2017 /Published online: 7 October 2017

(C) Springer-Verlag GmbH Germany 2017

\begin{abstract}
Aims/hypothesis Obesity and type 2 diabetes are concomitant with low-grade inflammation affecting insulin sensitivity and insulin secretion. Recently, the thioredoxin interacting protein (TXNIP) has been implicated in the activation process of the NOD-like receptor family, pyrin domain containing 3 (NLRP3) inflammasome. In this study, we aim to determine whether the expression of TXNIP is altered in the circulating immune cells of individuals with type 2 vs type 1 diabetes and whether this can be related to specific causes and consequences of inflammation.

Methods The expression of TXNIP, inflammatory markers, markers of the unfolded protein response (UPR) to
\end{abstract}

Alain Ktorza passed away just before the submission of this paper. This work is dedicated to his memory.

Electronic supplementary material The online version of this article (https://doi.org/10.1007/s00125-017-4462-5) contains peer-reviewed but unedited supplementary material, which is available to authorised users.

Fabienne Foufelle

fabienne.foufelle@crc.jussieu.fr

1 Inserm, UMRS 1138, Sorbonne Universités, UPMC Univ Paris 06; Sorbonne Paris Cité, Université Paris Descartes, Université Paris Diderot; Centre de Recherche des Cordeliers, 15 rue de l'école de médicine, 75006 Paris, France

2 Institut de Recherches Servier, Suresnes, France

3 Department of Endocrinology, Nutrition, and Diabetes, Pitié-Salpêtrière Hospital, Assistance Publique-Hôpitaux de Paris, Paris, France

4 Department of Diabetes and Endocrinology, Lariboisière Hospital, DHU FIRE, Assistance Publique-Hôpitaux de Paris, Paris, France

5 Department of Oncology and Endocrine Biochemistry, Pitié-Salpêtrière Hospital, Assistance Publique-Hôpitaux de Paris, Paris, France endoplasmic reticulum (ER) stress and enzymes involved in sphingolipid metabolism was quantified by quantitative reverse transcription real-time PCR (qRT-PCR) in peripheral blood mononuclear cells (PBMCs) of 13 non-diabetic individuals, 23 individuals with type 1 diabetes and 81 with type 2 diabetes. A lipidomic analysis on the plasma of 13 nondiabetic individuals, 35 individuals with type 1 diabetes and 94 with type 2 diabetes was performed. The effects of ER stress or of specific lipids on TXNIP and inflammatory marker expression were analysed in human monocyte-derived macrophages (HMDMs) and THP-1 cells.

Results The expression of TXNIP and inflammatory and UPR markers was increased in the PBMCs of individuals with type 2 diabetes when compared with non-diabetic individuals or individuals with type 1 diabetes. TXNIP expression was significantly correlated with plasma fasting glucose, plasma triacylglycerol concentrations and specific UPR markers. Induction of ER stress in THP-1 cells or cultured HMDMs led to increased expression of UPR markers, TXNIP, NLRP3 and IL-1 $\beta$. Conversely, a chemical chaperone reduced the expression of UPR markers and TXNIP in PBMCs of individuals with type 2 diabetes. The lipidomic plasma analysis revealed an increased concentration of saturated dihydroceramide and sphingomyelin in individuals with type 2 diabetes when compared with non-diabetic individuals and individuals with type 1 diabetes. In addition, the expression of specific enzymes of sphingolipid metabolism, dihydroceramide desaturase 1 and sphingomyelin synthase 1 , was increased in the PBMCs of individuals with type 2 diabetes. Palmitate or C2 ceramide induced ER stress in macrophages as well as increased expression of TXNIP, NLRP3 and IL-1 $\beta$.

Conclusions/interpretation In individuals with type 2 diabetes, circulating immune cells display an inflammatory phenotype that can be linked to ER stress and TXNIP expression. 
Immune cell ER stress can in turn be linked to the specific exogenous and endogenous lipid environment found in type 2 diabetes.

Keywords Ceramide $\cdot$ Diabetes mellitus $\cdot$ Endoplasmic reticulum stress · Inflammasome · Macrophages · NLRP3 . Peripheral blood mononuclear cells $\cdot$ Sphingolipids $\cdot$ TXNIP

$\begin{array}{ll}\text { Abbreviations } \\ \text { ATF6 } & \text { ER stress activated transcription factor } 6 \\ \text { BMDM } & \text { Bone marrow-derived macrophages } \\ \text { CCL2 } & \text { Chemokine ligand 2 } \\ \text { ChREBP } & \text { Carbohydrate response element binding protein } \\ \text { ER } & \text { Endoplasmic reticulum } \\ \text { HMDM } & \text { Human monocyte-derived macrophage } \\ \text { IRE1 } & \text { Inositol requiring enzyme 1 } \\ \text { NLRP3 } & \text { NOD-like receptor family, pyrin domain } \\ & \text { containing 3 } \\ \text { PBA } & \text { 4-Phenylbutyric acid } \\ \text { PBMC } & \text { Peripheral blood mononuclear cell } \\ \text { PERK } & \begin{array}{l}\text { Protein kinase R-like endoplasmic reticulum } \\ \text { kinase }\end{array} \\ \text { qRT-PCR } & \text { Quantitative reverse transcription real-time PCR } \\ \text { TLR } & \text { Toll-like receptor } \\ \text { TUDCA } & \text { Tauroursodeoxycholic acid } \\ \text { TXNIP } & \text { Thioredoxin interacting protein } \\ \text { UPR } & \text { Unfolded protein response } \\ \text { XBP1 } & \text { X box binding protein 1 }\end{array}$

\section{Introduction}

Obesity is concomitant with alterations in lipid environment and with chronic low-grade inflammation, which contributes to insulin resistance and paves the way to type 2 diabetes [1]. The inflammation originates from adipose tissue upon macrophage infiltration. Macrophages secrete inflammatory cytokines such as chemokine ligand 2 (CCL2), TNF $\alpha$ and IL$1 \beta$. These cytokines participate locally in insulin resistance of adipose tissue but can also have a systemic role in modulating insulin sensitivity of liver and muscles. Circulating monocytes also present an enhanced inflammatory phenotype [2]. Inflammation is involved in the decrease of insulin secretion [3]. Indeed, an increased number of macrophages and inflammation markers such as IL-1 $\beta$ are detected in the pancreatic islets of individuals with type 2 diabetes [4].

The NOD-like receptor family, pyrin domain containing 3 (NLRP3) inflammasome has a key role in the regulation of immunity and inflammation [5]. The inflammasome is formed from NLRP3, apoptosis-associated speck-like protein (ASC) and procaspase 1 . When stimulated, it promotes the activation, by a caspase-1-dependent mechanism, of procytokines such as pro-IL-1 $\beta$ or pro-IL-18 into biologically active cytokines. The NLRP3 inflammasome is activated in a sequential process requiring a priming event that mainly increases the expression of NLRP3 and an activation process leading to inflammasome assembly. Several signals have been described for NLRP3 inflammasome activation including microbial products or endogenous molecules (e.g. ATP).

Recently, thioredoxin interacting protein (TXNIP) has been implicated in the activation of the NLRP3 inflammasome in the presence of endoplasmic reticulum (ER) stress [6, 7] or reactive oxygen species [8]. TXNIP was originally described as an inhibitor of thioredoxin [9] and thus is a cellular redox regulator. TXNIP was also found to be the gene most potently induced by glucose in pancreatic beta cells by a mechanism involving the transcription factor carbohydrate response element binding protein (ChREBP) [10]. TXNIP is described as a pro-apoptotic factor favouring, when overexpressed, the death of pancreatic beta cells.

In the present study, we aim to determine whether circulating immune cells of individuals with type 2 diabetes (compared with non-diabetic individuals or individuals with type 1 diabetes) have an altered expression of TXNIP that can be related to inflammation and to alterations in the lipid environment.

\section{Methods}

Clinical cohorts Blood samples were obtained, over a 15 month period, from 281 individuals with type 1 diabetes ( $n=69)$ or type 2 diabetes $(n=212)$ hospitalised in the diabetes departments of the Lariboisière and the Pitié-Salpêtrière Hospitals (Paris, France). Non-diabetic individuals $(n=13$; fasting glycaemia $<6.9 \mathrm{mmol} / \mathrm{l}$ ), as a control group, were recruited in the vascular surgery departments of the PitiéSalpêtrière Hospital; these individuals were suffering from vascular diseases unrelated to atheromatous diseases (e.g. Ehlers Danlos disease), were not being treated with glucoselowering drugs and did not have a history of diabetes, metabolic syndrome or metabolic diseases. Individuals with infectious or immune pathology, with anti-inflammatory or immune suppressive therapy were excluded.

Ethics During hospitalisation, written consent was obtained from each individual and anthropometric and biological characteristics were recorded. Ethical approval for the use of human plasma was given by the Ethics Committee of the PitiéSalpêtrière hospital (CPP Ile de France VI - Paris, France).

Isolation of human peripheral blood mononuclear cells Human peripheral blood mononuclear cells (PBMCs) were 
isolated by Ficoll density gradient centrifugation (GE Healthcare Bio-Sciences, Uppsala, Sweden). PBMCs were collected and washed in PBS without $\mathrm{CaCl}_{2}$ and $\mathrm{MgCl}_{2}(1 \times)$ with 2\% heat-inactivated FBS (Gibco Thermo Fisher Scientific, Waltham, MA, USA) and $2 \mathrm{mmol} / \mathrm{l} \mathrm{EDTANa}_{2}$ at $650 \mathrm{~g}$ for $10 \mathrm{~min}$ at $4^{\circ} \mathrm{C}$. Then PBMCs were washed twice with red blood cells lysis buffer (Sigma-Aldrich, St Louis, MO, USA). Finally, the cells were resuspended in PBS with $2 \% \mathrm{FBS}, 2 \mathrm{mmol} / \mathrm{LDTANa} \mathrm{Ea}_{2}$ and counted; $1 \times 10^{6}$ cells were used for RNA studies.

Animals Wistar rats were purchased from Charles River Laboratories (St Germain Nuelle, France). They were housed under a $12 \mathrm{~h}$ light-dark cycle in a temperature-controlled environment and had free access to water and regular diet $(65 \%$ carbohydrate, $11 \%$ fat, $24 \%$ protein). All animal care and experimental procedures were conducted in accordance with the French Law of 6 April 2010, and they were approved by the local ethics committee (Charles Darwin, Ce5/2012/052). Primary rat hepatocytes were isolated as described previously [11].

Cell culture and treatment Cell cultures were tested for the absence of mycoplasma using the Universal mycoplasma detection kit (ATCC, Manassas, VA, USA). The THP-1 monocytic cell line (ATCC) was seeded at $0.5 \times 10^{6}$ cells $/ \mathrm{ml}$ in RPMI 1640 supplemented with $10 \%$ heat-inactivated FBS and $1 \%$ penicillin streptomycin (Gibco Thermo Fisher Scientific). The cells were differentiated at $1 \times 10^{6}$ cells $/ \mathrm{ml}$ in the presence of $1 \mu \mathrm{g} / \mathrm{ml}$ of phorbol 12-myristate 13-acetate (Merck Millipore, Darmstadt, Germany) for $72 \mathrm{~h}$. Then they were pre-treated overnight in medium with or without $1 \mathrm{mmol} / \mathrm{l}$ tauroursodeoxycholic acid (TUDCA) (Merck Millipore) or $10 \mu \mathrm{mol} / \mathrm{l}$ myriocin (Enzo life, New York, NY, USA) and incubated as follows: (1) with ER stress inducers $300 \mathrm{nmol} / 1$ thapsigargin (Merck Millipore) or $1 \mathrm{ng} / \mathrm{ml}$ tunicamycin (Merck Millipore) for $24 \mathrm{~h}$; (2) with $0.25 \mathrm{mmol} / \mathrm{l}$ palmitate conjugated with fatty acid-free BSA (Sigma-Aldrich) for $24 \mathrm{~h}$ as described in [12]; or (3) with $50 \mu \mathrm{mol} / \mathrm{l} \mathrm{C} 2$-ceramide (Merck Millipore) for $6 \mathrm{~h}$.

PBMCs were cultured in suspension at $2 \times 10^{6}$ cells $/ \mathrm{ml}$ for $24 \mathrm{~h}$ in a complete medium containing RPMI medium 1640 , $1 \%$ penicillin streptomycin, $1 \%$ Minimum Essential Medium (MEM) Non-Essential Amino Acids (NEAA) (100×, Gibco Thermo Fisher Scientific) and 10\% human serum AB male HIV tested (Dutscher, Brumath, France) with or without $50 \mu \mathrm{mol} / 1$ 4-phenylbutyric acid (PBA) (Merck Millipore) for $24 \mathrm{~h}$.

Human monocyte-derived macrophages (HMDMs) were obtained from healthy volunteer donors (Don du Sang, Hôpital de la Pitié-Salpêtrière). PBMCs were isolated as described above and differentiated at $1 \times 10^{6}$ cells $/ \mathrm{ml}$ in complete PBMC medium supplemented with $100 \mathrm{ng} / \mathrm{ml}$ human M-CSF (Miltenyi Biotec, Bergisch Gladbach, Germany) for approximately 10 days. The cells were then treated with the ER stress inducers as described for THP-1 cells.

The samples were then processed for RNA extraction and protein analysis.

PBMCs, primary rat hepatocytes and immortalised mouse myoblast cell line $\mathrm{C} 2 \mathrm{C} 12$ were treated with low glucose $(5 \mathrm{mmol} / \mathrm{l})$ or high glucose $(25 \mathrm{mmol} / \mathrm{l})$ for $24 \mathrm{~h}$. The cells were then processed for RNA extraction and measurement of mRNA expression by quantitative reverse transcription realtime PCR (qRT-PCR).

SiRNA experiments Differentiated THP-1 cells were transfected with $25 \mathrm{nmol} / 1$ TXNIP siRNA (s20880; Ambion, Waltham, MA, USA) or scramble siRNA using lipofectamine RNAiMAX transfection reagent (Gibco Thermo Fisher Scientific). The cells were then treated with the ER stress inducers as described above. The sequences used are given in electronic supplementary material (ESM) Table 1.

Gene expression analysis PBMCs, HMDMs and THP- 1 cells were processed for total RNA extraction using the RNeasy Mini Kit (Qiagen, Venlo, the Netherlands). Complementary DNAs were prepared using $250 \mathrm{ng}$ of RNA with SuperScript II Reverse Transcriptase (Thermo Fisher Scientific). Mix Mesa Green qPCR MasterMix Plus (Eurogentec, Brussels, Belgium) was used for qRT-PCR using CFX384 (384-well plates) and an iQ5 system (Bio-Rad, Hercules, CA, USA). Ribosomal RNA 18S and $\beta$-actin were used for normalisation and for relative quantification of gene expression using the $2^{-\triangle \Delta \mathrm{C}_{\mathrm{t}}}$ method. The following genes were analysed: ATF4, CCL2, CERS2, CERS4, CHOP (also known as DDIT3), DES1 (DEGS1), ERP72 (PDIA4), HEDJ (DNAJB11), IL-1 $\beta$ (IL1B), NLRP3, P58IPK, SMS1 (SGMS1), TXNIP and XBP1. All samples were run in duplicate and the result was discarded when a difference higher than $0.2 C_{t}$ was observed between the duplicates. When the samples were not analysed in a single run, an internal control was used to account for qRT-PCR inter-variability. Results are from three independent experiments quantified in triplicate. For analysis of $X B P 1 \mathrm{mRNA}$ splicing, $10 \mathrm{ng}$ cDNA was amplified with specific primers for human XBP1. The conditions used for the qRT-PCR were described previously [11]. PCR products were separated by electrophoresis on $2 \%$ low-melting agarose gels, visualised by ethidium bromide staining and quantified by densitometric analysis of spliced $X B P 1$ and unspliced $X B P 1$. The results are shown as the ratio of spliced or unspliced $X B P 1 \mathrm{mRNA}$ to total $X B P 1 \mathrm{mRNA}$ (spliced $X B P 1+$ unspliced $X B P 1$ ). The primers used are given in ESM Table 1.

Western blotting and protein analysis HMDM and THP-1 cells were washed twice in cold PBS, scraped in RIPA lysis buffer (Sigma-Aldrich) and complete protease inhibitor cocktail (Roche diagnostics, Mannheim, Germany) and 
centrifuged $\left(10,000 \mathrm{~g}, 10 \mathrm{~min}, 4^{\circ} \mathrm{C}\right)$. Twenty micrograms of protein samples were subjected to SDS-PAGE. After electrophoresis, proteins were transferred to a nitrocellulose membrane. The primary antibodies used were anti-TXNIP (K02053; MLB International, Woburn, MA, USA) at a 1/1000 dilution, anti-NLRP3 (NBP2-12446; Novus Biologicals, Littleton, CO, USA) at a $1 / 750$ dilution and anti- $\beta$-actin (A5316; Sigma-Aldrich) at a 1/4000 dilution. The secondary antibodies were conjugated to horseradish peroxidase. The detection was performed with West Pico chemiluminescent substrate and the membranes were imaged with a MyECL Imager (Thermo Fisher Scientific). Characterisation was based on the protein molecular mass.

IL-1 $\beta$ ELISA IL-1 $\beta$ protein was quantified in the culture media of THP-1 cells by using a cytokine-specific ELISA kit (R\&D Systems, Minneapolis, MN, USA).

Plasma lipid analysis Three-hundred microlitres of plasma were used to quantify dihydroceramides, ceramides, sphingomyelins and sphingosine content. The lipid subspecies were extracted and analysed, as described in [13], at the Lipidomic Core Facility of the University of Bourgogne (Dijon, France). Plasma NEFA concentrations were determined using a colorimetric method (Randox Laboratories, Antrim, UK).

Blinding and statistical analysis Experimenters were blind to group assignment. All statistical analyses are presented as means \pm SEM or SD as indicated. No statistical method was used to predetermine sample size. Gaussian distribution was tested using the Kolmogorov-Smirnov test. Unpaired or paired Student's $t$ test and one-way (Dunn's post-test) or two-way (Bonferroni's post hoc test) ANOVA were used for comparison. $p<0.05$ was considered significant.

\section{Results}

Clinical characteristics The clinical and biochemical characteristics of the individuals are presented in Table 1. Plasma glucose and $\mathrm{HbA}_{1 \mathrm{c}}$ were higher in individuals with type 1 diabetes than in those with type 2 diabetes. Triacylglycerol concentrations were higher in individuals with type 2 diabetes, as was the total/HDL-cholesterol ratio.

Increased expression of TXNIP and inflammatory markers in PBMCs from individuals with type 2 diabetes Compared with TXNIP mRNA levels in PBMCs from nondiabetic individuals, levels were similar in individuals with type 1 diabetes and significantly higher in individuals with type 2 diabetes (Fig. 1a).

Since insulin has been described as decreasing TXNIP expression $[14,15]$, we separately analysed PBMCs from individuals with type 2 diabetes with $(n=20)$ or without $(n=61)$ insulin treatment. TXNIP expression was similar in individuals with type 2 diabetes irrespective of insulin treatment (results not shown).

In the type 2 diabetes group, TXNIP mRNA expression was significantly $(p<0.05)$ correlated with blood glucose and plasma triacylglycerols (Table 2). The fact that the individuals with type 1 diabetes had higher $\mathrm{HbA}_{1 \mathrm{c}}$ values than individuals with type 2 diabetes suggests that blood glucose is not the primary cause of the increased TXNIP expression in
Table 1 Clinical characteristics of the diabetic and non-diabetic individuals

\begin{tabular}{llll}
\hline Characteristic & $\begin{array}{l}\text { Non-diabetic } \\
\text { individuals }(n=13)\end{array}$ & $\begin{array}{l}\text { Individuals with } \\
\mathrm{T} 1 \mathrm{D}(n=23)\end{array}$ & $\begin{array}{l}\text { Individuals with } \\
\mathrm{T} 2 \mathrm{D}(n=81)\end{array}$ \\
\hline Age, years & $67.4 \pm 3.5$ & $39 \pm 13.8^{* * *}$ & $62.8 \pm 8.5^{\dagger \dagger \dagger}$ \\
$\mathrm{Sex}, n$ male/ $n$ female & $8 / 5$ & $11 / 12$ & $55 / 26$ \\
$\mathrm{BMI}, \mathrm{kg} / \mathrm{m}^{2}$ & $22 \pm 2.2$ & $22.9 \pm 2.1$ & $29.2 \pm 5.3^{* * *, \dagger \dagger \dagger}$ \\
Diabetes duration, years & - & $14.6 \pm 14$ & $12.4 \pm 8.0$ \\
Fasting plasma glucose, mmol/1 & $4.83 \pm 0.36$ & $11.8 \pm 4.7^{* * *}$ & $8.4 \pm 2.5^{* * *, \dagger \dagger}$ \\
$\mathrm{HbA}_{1 \mathrm{c}}, \%$ & - & $8.8 \pm 2.4$ & $7.75 \pm 1.42^{\dagger \dagger \dagger}$ \\
$\mathrm{HbA}_{1 \mathrm{c}}, \mathrm{mmol} / \mathrm{mol}$ & - & $72 \pm 19$ & $61 \pm 11^{\dagger \dagger}$ \\
Plasma triacylglycerol, mmol/1 & $1.33 \pm 0.5$ & $0.92 \pm 0.40^{* *}$ & $1.83 \pm 0.96^{\dagger \dagger \dagger}$ \\
Plasma NEFA, mmol/1 & $0.29 \pm 0.23$ & $0.35 \pm 0.20$ & $0.30 \pm 0.13$ \\
Plasma total cholesterol, mmol/1 & $46 \pm 10$ & $50 \pm 10$ & $43 \pm 11$ \\
Plasma LDL-cholesterol, mmol/1 & $27 \pm 9$ & $29 \pm 7$ & $23 \pm 6^{*, \dagger \dagger \dagger}$ \\
Plasma HDL-cholesterol, mmol/1 & $15 \pm 3$ & $17 \pm 5$ & $11 \pm 4^{\dagger}$ \\
Plasma total cholesterol/HDL-cholesterol ratio & $3.0 \pm 0.9$ & $3.1 \pm 0.8$ & $4.09 \pm 1.5^{* *, \dagger \dagger \dagger}$ \\
\hline
\end{tabular}

Results are presented as mean \pm SD unless stated otherwise

$* p<0.05, * * p<0.01$ and $* * * p<0.001$ vs non-diabetic; ${ }^{\dagger} p<0.05$ and ${ }^{\dagger \dagger} p<0.001$, T2D vs T1D

T1D, type 1 diabetes; T2D, type 2 diabetes 
Fig. $1 T X N I P$ and inflammatory genes are increased in PBMCs from individuals with type 2 diabetes. Scatter plots show the relative expression of TXNIP (a), $N L R P 3(\mathbf{b}), I L-1 \beta$ (c) and CCL2 (d) mRNA in PBMCs from nondiabetic individuals (control, $n=13$ ), individuals with type 1 diabetes (T1D, $n=23$ ) and individuals with type 2 diabetes (T2D, $n=81$ ). mRNA levels were quantified by qRT-PCR. $* p<0.05, * * p<0.01$ and $* * * p<0.001$ for indicated comparisons. Horizontal bars indicate the mean value \pm SEM. AU, arbitrary units
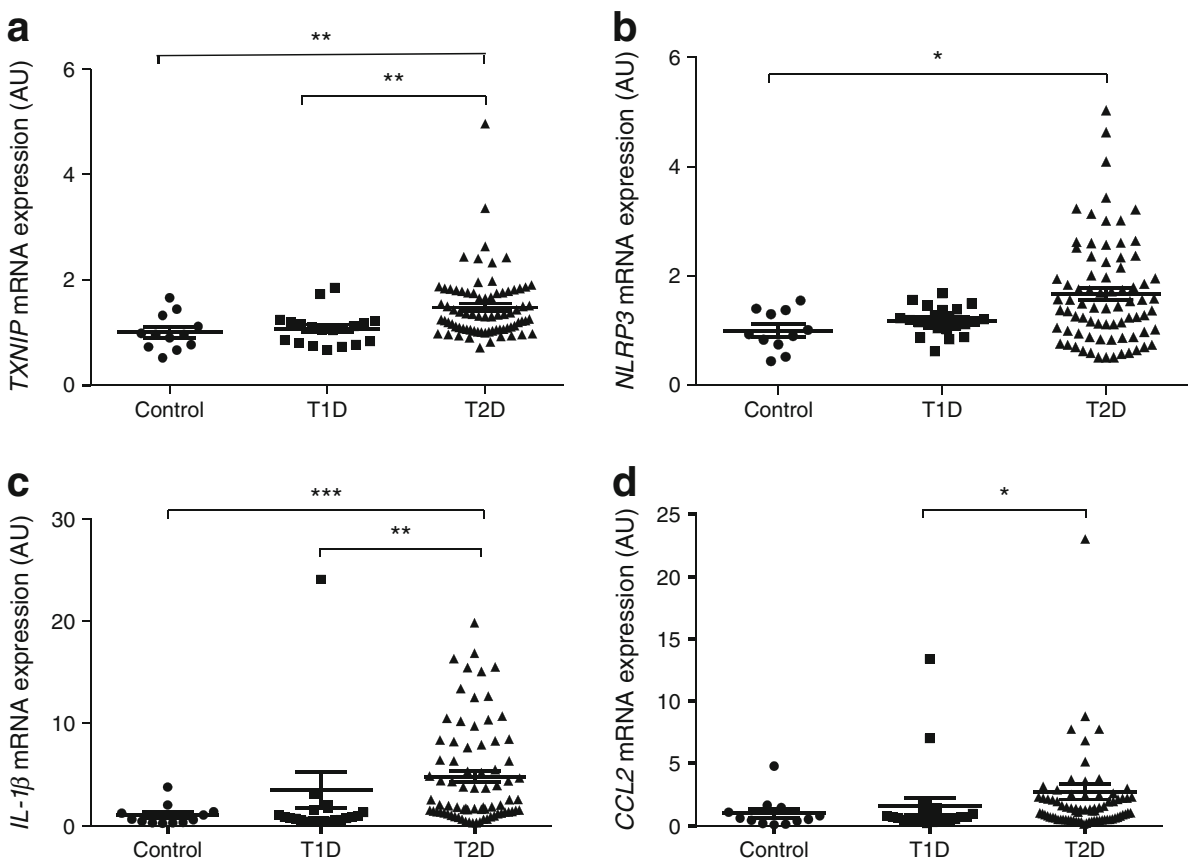

type 2 diabetes. However, and in order to exclude the fact that PBMCs from individuals with type 1 and type 2 diabetes could respond differently to the prevailing hyperglycaemia, we cultured PBMCs from both groups of individuals in the presence of $5 \mathrm{mmol} / 1$ or $25 \mathrm{mmol} / 1$ glucose. A high glucose concentration was not able to increase levels of TXNIP mRNA in PBMCs from individuals with either type 1 or type 2 diabetes (ESM Fig. 1) whereas there was a clear effect of glucose on Txnip expression in primary cultured rat hepatocytes or C2C12 mouse muscle cells, as shown previously [16, 17].

Expression of $N L R P 3$ (Fig. 1b) and $I L-1 \beta$ (Fig. 1c) was significantly increased in the PBMCs of individuals with type 2 diabetes compared with the control group. CCL2 expression levels were higher in PBMCs from individuals with type 2 diabetes compared with type 1 diabetes (Fig. 1d). The differences we observed between the groups for these variables (TXNIP, NLRP3, IL1- $\beta$, CCL2) were similar whatever the sex of the individual (results not shown).

\section{Increased expression of unfolded protein response} markers in PBMCs from individuals with type 2 diabetes In pancreatic beta cells, TXNIP is a link between ER stress and inflammation $[6,7]$. We thus examined the expression of unfolded protein response (UPR) markers related to the protein kinase R-like endoplasmic reticulum kinase (PERK), ER stress activated transcription factor 6 (ATF6) or inositol requiring enzyme (IRE1)/ X-box binding protein 1 (XBP1) UPR branch. The mRNA expression of UPR markers downstream of PERK (ATF4 and CHOP [transcription factors]) and IRE1/XBP1 (HEDJ [luminal chaperone]) and the mRNA expression of P58IPK (a protein involved in a feedback inhibition of the UPR [18]) were found to be selectively elevated in
PBMCs from individuals with type 2 diabetes (Fig. 2a-d). In addition, XBP1 mRNA splicing, a marker of IRE1 activation, was greater in PBMCs from individuals with type 2 diabetes than in PBMCs from individuals with type 1 diabetes (Fig. 2e). In contrast, the mRNA expression of two target genes of ATF6, ERP72 (encoding protein disulfide isomerase family A member 4 [19]) and XBP1 [20], was similar among the three groups of individuals (Fig. 2f,g). TXNIP mRNA expression in PBMCs of individuals with type 2 diabetes was significantly positively correlated with expression of P58IPK and ATF4 mRNA (Table 2).

Induction of ER stress in macrophages promotes TXNIP expression To address the question of whether there is a causal relationship between ER stress and TXNIP expression in

Table 2 Correlations between TXNIP expression in PBMCs of individuals with type 2 diabetes and clinical variables

\begin{tabular}{lll}
\hline Variable & $r_{\mathrm{s}}$ & $\begin{array}{l}p \text { value } \\
\text { (vs PBMC TXNIP mRNA) }\end{array}$ \\
\hline BMI & 0.05 & 0.66 \\
Age & 0.20 & 0.09 \\
Plasma glucose & 0.30 & 0.014 \\
Plasma triacylglycerol $_{\text {HbA }_{1 \mathrm{c}}}$ & 0.30 & 0.010 \\
PBMC P58IPK mRNA $_{\text {PBMC ATF4 mRNA }}$ & 0.19 & 0.13 \\
PBMC NLRP3 mRNA & 0.43 & 0.0002 \\
PBMC DES1 mRNA & 0.11 & 0.05 \\
PBMC SMS1 mRNA & 0.24 & 0.35 \\
\hline
\end{tabular}


Fig. 2 UPR markers are increased in PBMCs from individuals with type 2 diabetes. (a-d, f, g) Scatter plots show the relative mRNA expression of UPR markers from PERK $(\mathbf{a}, \mathbf{b})$, IRE1 (c, d) and ATF6 (f, g) pathways in PBMCs from nondiabetic individuals (control, $n=13$ ), individuals with type 1 diabetes (T1D, $n=23)$ and individuals with type 2 diabetes (T2D, $n=81$ ). mRNA levels were quantified by qRT-PCR. ${ }^{*} p<0.05, * * p<0.01$ and $* * * p<0.001$ for indicated comparisons. Horizontal bars indicate the mean value \pm SEM. AU, arbitrary units. (e) IRE1 activation was assessed by the quantification of XBP1 splicing. Unspliced and spliced forms of XBP1 mRNA were amplified by qRT-PCR followed by gel running and densitometric quantification. The representative blot shows the expression of unspliced $X B P 1$ (uXBP1; $424 \mathrm{bp})$ and spliced $X B P 1$ (sXBP1; 398 bp) in PBMCs from individuals with type 1 diabetes (T1D, $n=6$ ) and type 2 diabetes (T2D, $n=6$ ).

The histogram represents the densitometric quantification of $\mathrm{s} X B P 1$ and $\mathrm{u} X B P 1$ in PBMCs from individuals with type 1 diabetes (white bars, $n=23$ ) and type 2 diabetes (black bars, $n=78$ ). The results are shown as the ratio of spliced or unspliced $X B P 1$ mRNA to total $X B P 1$ mRNA $(\mathrm{s} X B P 1+\mathrm{u} X B P 1)$.

Results (and horizontal bars in scatter plots) are mean \pm SEM. ${ }_{* * * * *} p<0.001$ vs type 1 diabetes
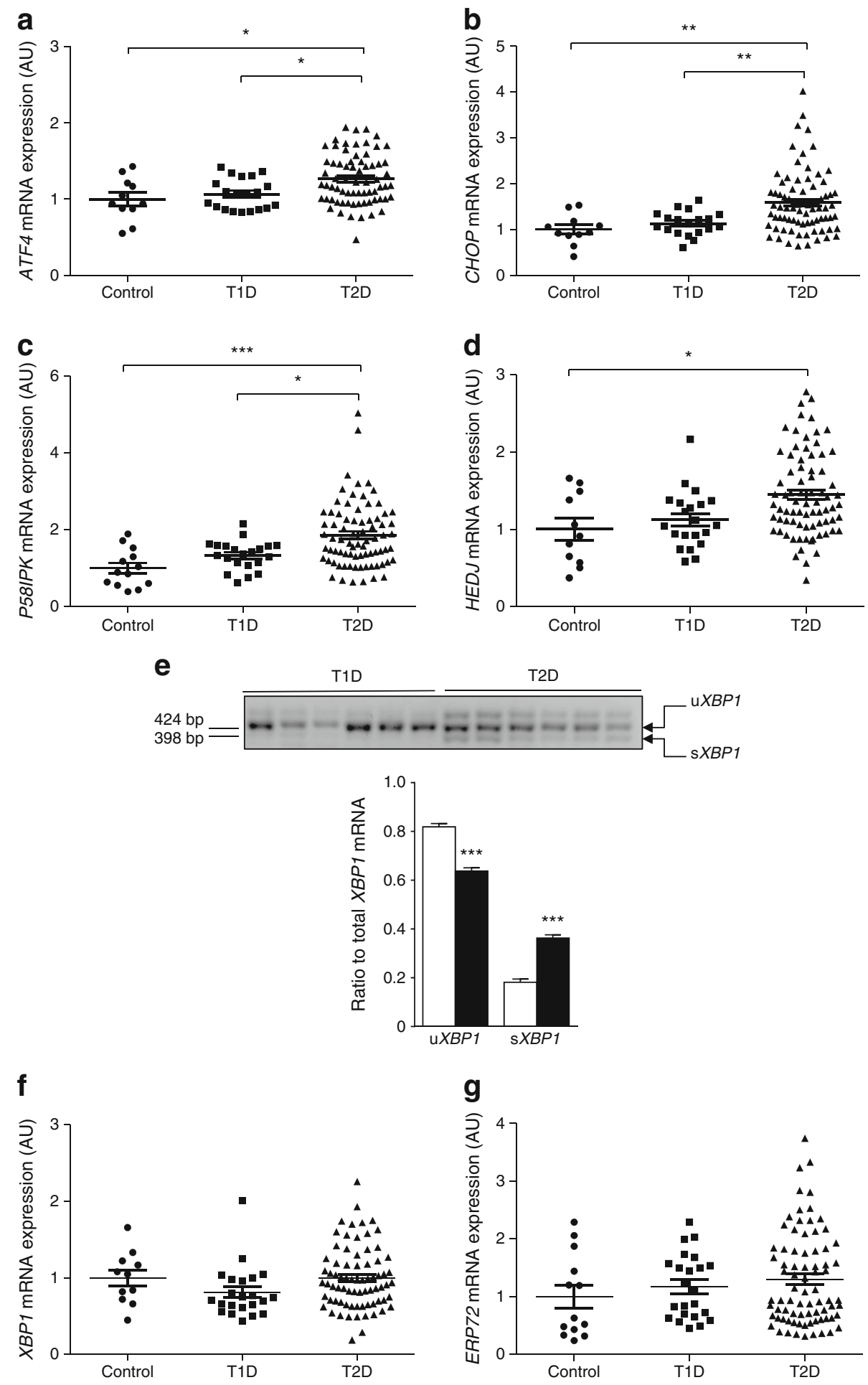

PBMCs, we performed in vitro experiments in a human macrophage cell line, THP-1. Macrophages were cultured in the presence of two ER stress inducers, thapsigargin and tunicamycin. Measurement of ATF4, P58IPK, HEDJ and ERP72 mRNA expression confirmed the induction of ER stress by the two compounds (Fig. 3a-d). This was concomitant with a large increase in TXNIP expression (Fig. 3e).
Culture of macrophages in the presence of TUDCA, a chemical chaperone that reduces ER stress (Fig. 3a,b), opposed the effect of ER stress inducers on expression of TXNIP mRNA (Fig. 3e) and TXNIP protein (Fig. 3g). Finally, in THP1 macrophages, IL-1 $\beta$ secretion was increased by thapsigargin alone but inhibited by the presence of thapsigargin and TUDCA (Fig. 3f). 
a

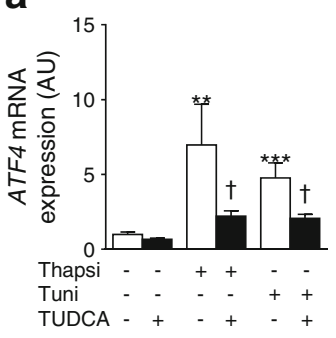

e

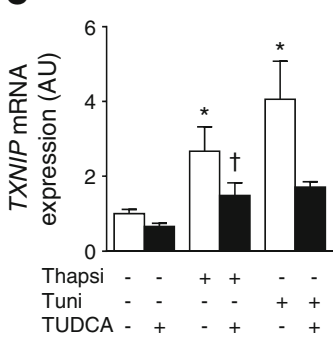

h

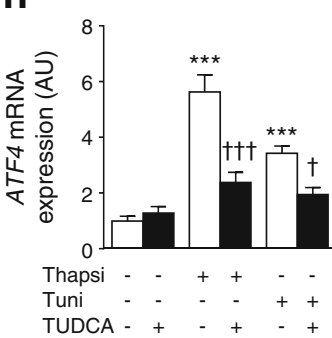

I

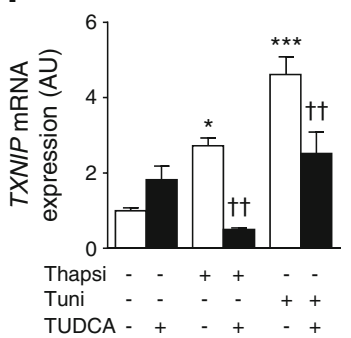

b

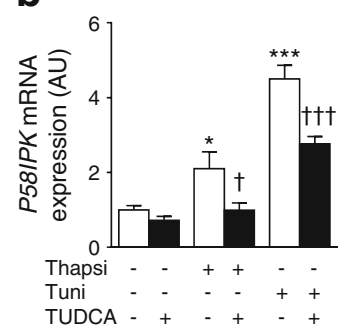

f

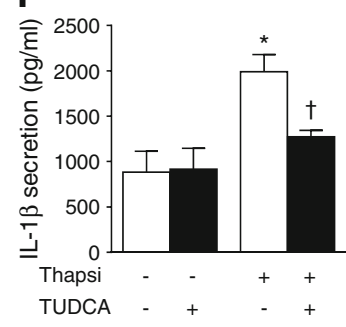

i

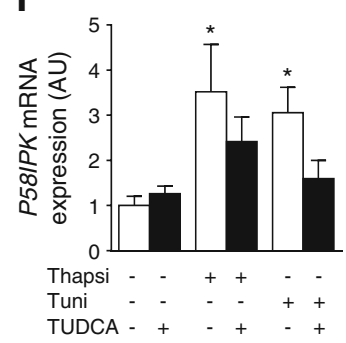

m

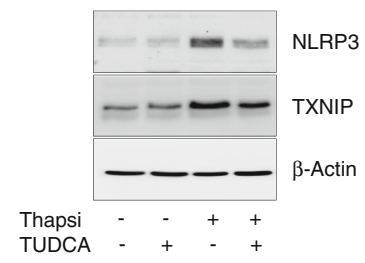

C

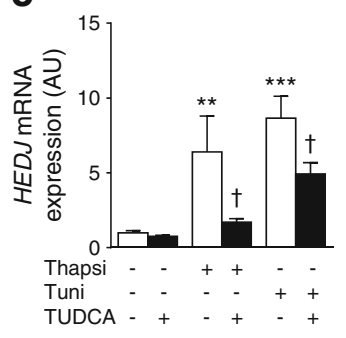

g d

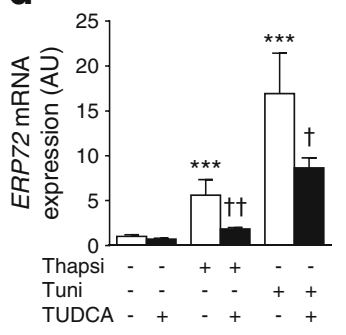

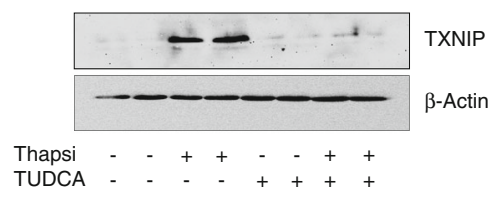

j
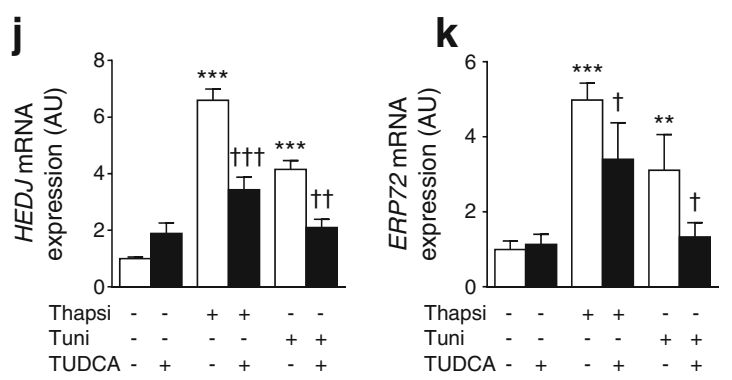

$* p<0.05, * * p<0.01$ and $* * * p<0.001$ relative to untreated cells; ${ }^{\dagger} p<0.05,{ }^{\dagger} p<0.01$ and ${ }^{\dagger \dagger} p<0.001$ relative to ER stress conditions (cells treated with Thapsi or Tuni without TUDCA). AU, arbitrary units. Cell lysates from THP-1 cells (g) and HMDMs (m) treated with Thapsi with or without TUDCA were immunoblotted with antibodies against NRLP3, TXNIP and $\beta$-actin 作 was used for the quantification of IL-1 $\beta$ secretion in the Thapsi-treated THP-1 cells. Results are mean \pm SEM for three independent experiments.

Similar results were obtained in cultured HMDMs. Thapsigargin and tunicamycin induced UPR markers and TXNIP mRNA expression (Fig. 3h-1), an effect counteracted by TUDCA. Interestingly, this was paralleled by changes in TXNIP and NLRP3 protein content (Fig. 3m).

We then tested whether decreasing the ER stress in PBMCs of a subset of individuals with type 2 diabetes (Table 3 ) was concomitant with an effect on TXNIP expression. PBMCs were cultured for $24 \mathrm{~h}$ in the absence or presence of a chemical

chaperone, PBA. We used PBA instead of TUDCA since TUDCA was toxic in these conditions. PBA induced a decrease in the expression of UPR markers and a consistent decrease in TXNIP mRNA expression (Fig. 4a-e).

Finally, to demonstrate a causal relationship between TXNIP and inflammation, we used a TXNIP siRNA in THP1 cells. Decreasing the expression of TXNIP (Fig. 4f) led to a decreased secretion of IL-1 $\beta$ even in the presence of ER stress inducers (Fig. 4h) whereas CHOP (Fig. 4g), an ER stress 
Table 3 Clinical characteristics of individuals with type 2 diabetes involved in the effects of PBA on PBMCs

\begin{tabular}{ll}
\hline Characteristic & Measurement $(n=23)$ \\
\hline Age, years & $60.5 \pm 10$ \\
Sex, $n$ male/ $n$ female & $10 / 13$ \\
BMI, $\mathrm{kg} / \mathrm{m}^{2}$ & $30.3 \pm 6$ \\
Diabetes duration, years & $16.0 \pm 9.5$ \\
$\mathrm{HbA}_{1 \mathrm{c}}, \%$ & $7.6 \pm 1.1$ \\
$\mathrm{HbA}_{1 \mathrm{c}}, \mathrm{mmol} / \mathrm{mol}$ & $60 \pm 9$ \\
Triacylglycerols, mmol/1 & $1.81 \pm 1.32$ \\
Total cholesterol, mmol/1 & $45 \pm 9$ \\
LDL-cholesterol, mmol/l & $26 \pm 8$ \\
HDL-cholesterol, $\mathrm{mmol} / \mathrm{l}$ & $12 \pm 3$ \\
Total/HDL-cholesterol ratio & $3.8 \pm 0.8$ \\
\hline
\end{tabular}

The results are presented as mean $\pm \mathrm{SD}$ unless stated otherwise

marker, was not affected, suggesting that TXNIP acts downstream of ER stress to promote inflammation.

Lipids, ER stress and TXNIP expression Next, we investigated the potential origin of ER stress in immune cells of individuals with type 2 diabetes. We reasoned that one main difference between type 1 and type 2 diabetes is the lipid environment and the degree of dyslipidaemia. Dyslipidaemia is more marked in type 2 diabetes due to the high prevalence of obesity and insulin resistance in this pathology as exemplified by the higher plasma triacylglycerol concentrations in individuals with type 2 diabetes or the higher total cholesterol/HDL-cholesterol ratio (Table 1). Another interesting lipid species is ceramide. The sphingolipid ceramides are closely related to inflammation [21] and are known to induce insulin resistance in muscles [22]. Ceramide can also induce ER stress [23].

Lipid species in monocytes/macrophages can originate endogenously through specific synthesis or exogenously from structures containing lipids, such as LDL [24]. Concerning the endogenous metabolism of sphingolipids, we found in PBMCs from individuals with type 2 diabetes an increased expression of dihydroceramide desaturase 1 (Fig. 5a), the enzyme that converts dihydroceramide into ceramide, and an increase in sphingomyelin synthase 1 (Fig. 5c), the enzyme that converts ceramide into sphingomyelin. Serine palmitoyl transferase 1 and ceramide synthase 2 and 4 expression was not increased (Fig. 5b,d and results not shown).

We then investigated, using a lipidomic approach, the plasma sphingolipid subspecies profiles of the various groups of individuals. For this study, the non-diabetic individuals forming the control group were the same as those described previously (Table 1) but owing to limited plasma availability, new groups of individuals with type 1 or type 2 diabetes were used (see Table 4). The new diabetic individuals had essentially similar profiles to those of the individuals described previously in Table 1.

Total circulating dihydroceramide concentrations were increased in the plasma of individuals with type 2 diabetes when compared with individuals with type 1 diabetes and the increase approached significance $(p<0.06)$ when compared with the control group (Fig. 6a). Total sphingomyelin concentrations were increased in the plasma of individuals with type 2 diabetes when compared with the two other groups (Fig. $6 \mathrm{~b}$ ), whereas total ceramide and sphingosine plasma concentrations were unchanged (Fig. 6c,d). Looking at subspecies of dihydroceramides, saturated forms were increased, whereas unsaturated forms were decreased (ESM Fig. 2). The details of the most abundant ceramide and sphingomyelin subspecies are given respectively in ESM Figs 3 and 4.

Thus, sphingolipid metabolism in immune cells of individuals with type 2 diabetes can be affected both endogenously and exogenously.

We then used the human macrophage cell line (THP-1) to test whether ceramides or their precursor, palmitate, could induce the sequence of ER stress, TXNIP expression and inflammation. In the presence of palmitate in the culture medium, we indeed observed an increased mRNA expression of UPR markers, ATF4, HEDJ and CHOP (Fig. 7a-c). Levels of TXNIP mRNA and TXNIP protein were also increased (Fig. $7 \mathrm{~d}, \mathrm{e}$ ), as were mRNA levels of inflammation markers $N L R P 3$ and $I L-1 \beta$ (Fig. 7f-h). These effects were fully reduced in the presence of myriocin (an inhibitor of ceramide synthesis from palmitate) and TUDCA (an inhibitor of ER stress) (Fig. 7a-h). Similar results were obtained for TXNIP protein in the absence or presence of myriocin. We then repeated the experiment in the presence of $\mathrm{C} 2$-ceramide in the culture medium and observed, as with palmitate, an induction of UPR markers (Fig. 8a-c), TXNIP (Fig. 8d,e) and inflammatory markers (Fig. 8 fh). TUDCA opposed the effects of ceramide on TXNIP mRNA, TXNIP protein and inflammatory markers (Fig. 8a-h).

\section{Discussion}

This study shows a concomitant increase in the expression of ER stress markers, TXNIP and inflammatory markers including NLRP3 in circulating immune cells of individuals with type 2 diabetes. This is specific to type 2 diabetes, since individuals with type 1 diabetes do not present such effects. The endogenous and exogenous lipid environment of immune cells in individuals with type 2 diabetes is also modified, especially concerning triacylglycerols, sphingolipid species and enzymes of sphingolipid metabolism. In vitro studies in macrophages suggest that the sequence that leads to this specific phenotype starts with a lipid-induced ER stress leading to TXNIP expression, which in turn activates inflammatory pathways. 
Fig. 4 Effects of a chemical chaperone and of a reduced expression of TXNIP on ER stress and inflammation markers. (a-e) PBMCs from individuals with type 2 diabetes were incubated with or without (control) PBA. mRNA levels were quantified by qRT-PCR. Results are presented for each individual $(n=21) . * p<0.05$, $* * p<0.01$ and $* * * p<0.001$ vs control. Horizontal bars indicate the mean value. AU, arbitrary units. (f-h) THP-1 cells were cultured with or without thapsigargin (Thapsi) or tunicamycin (Tuni) in the presence of TXNIP siRNA (si $T X N I P$ ) or a scramble siRNA (si scramble). mRNA levels were quantified by qRT-PCR. Medium was used for the quantification of IL-1 $\beta$ secretion. Results are mean \pm SEM for three independent experiments. $* * p<0.01$ and $* * * p<0.001$ vs si scrambletreated cells; ${ }^{\dagger} p<0.05$ vs ER stress conditions. AU, arbitrary units a

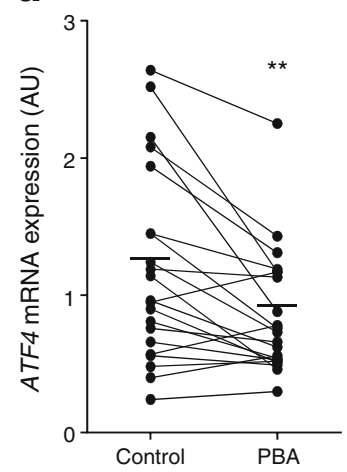

b

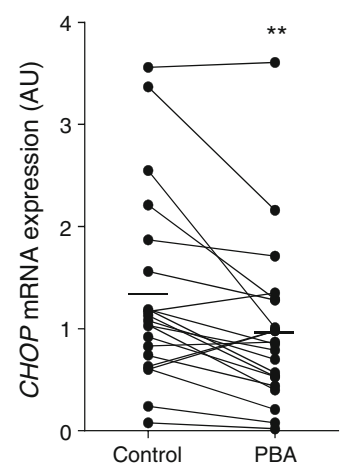

C

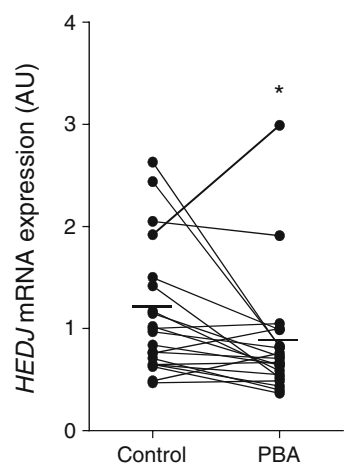

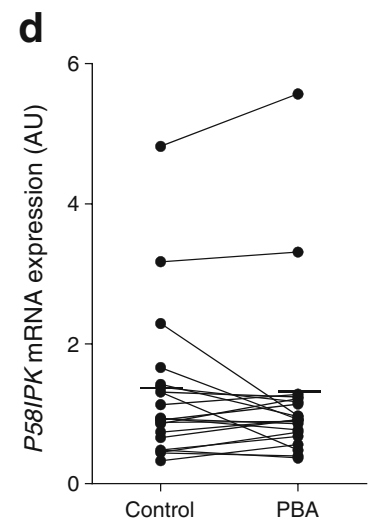
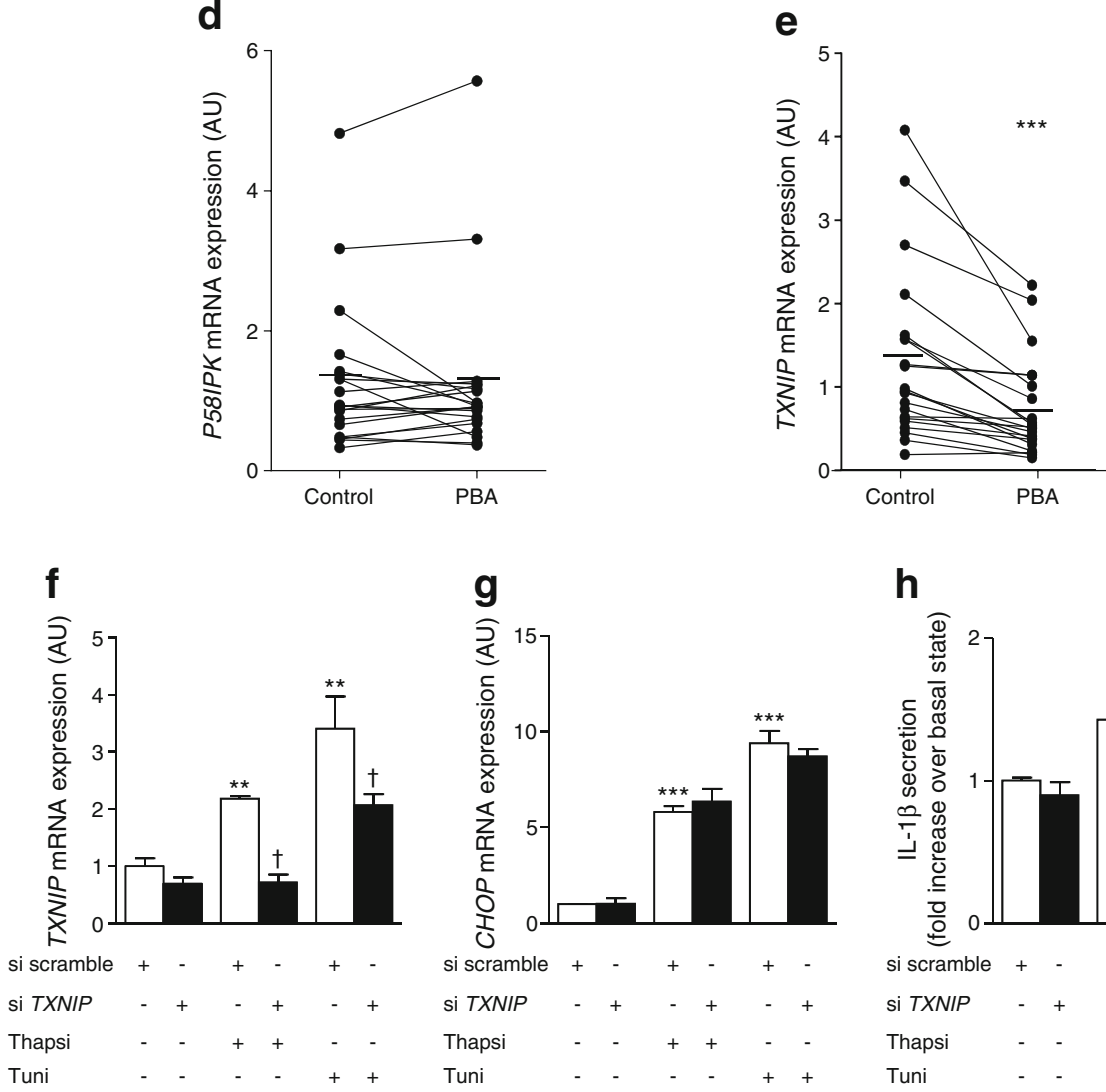

h

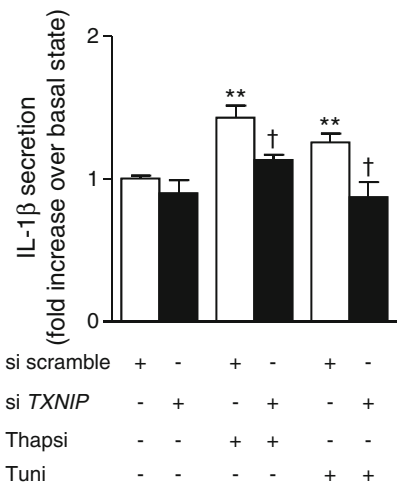

The strengths of this study include the comparison of a large cohort of individuals with type 2 diabetes with nondiabetic individuals and individuals with type 1 diabetes, allowing the exclusion of a specific role for glycaemia in the observed phenotypes, and the simultaneous measure of exogenous lipid environment and endogenous alterations in enzymes involved in sphingolipid metabolism. In addition, we were able to establish in vitro a causal link in macrophages between alterations in the lipid environment, ER stress, TXNIP and inflammation. One weakness is that, for reasons of material availability, we did not measure sphingolipid species in the PBMCs of the various groups.

The triad of abnormal lipid metabolism, ER stress and inflammation is found in metabolic diseases [25]. In pancreatic beta cells fatty acids induce ER stress, which leads to inflammation, secretory dysfunction and apoptosis $[6,7,26-28]$. In the liver, ER stress is present in the case of obesity and dysregulation of hepatic lipid metabolism (steatosis) $[11,29,30]$ and is concomitant with the induction of inflammatory pathways [31]. In inflammatory macrophages, lipotoxic signals (such as an excess of cholesterol metabolism) induce ER stress that can lead to lipotoxic death, thus contributing to the formation of atherosclerotic plaques $[32,33]$. Interestingly, saturated fatty acids induce, in mouse bone marrow-derived macrophages (BMDMs), the expression of genes related to ER stress and UPR and an inflammation that is dependent upon IRE $1 \alpha$ activation [34]. 
Fig. 5 Increased expression of enzymes involved in de novo sphingolipid synthesis in PBMCs from individuals with type 2 diabetes. Scatter plots show the relative expression of enzyme mRNA in PBMCs from nondiabetic individuals (control, $n=13$ ), individuals with type 1 diabetes (T1D, $n=23$ ) and individuals with type 2 diabetes (T2D, $n=81$ ). mRNA levels were quantified by qRT-PCR.

Horizontal bars indicate the mean value \pm SEM. $* p<0.05$,

$* * p<0.01$ and $* * * p<0.001$ for indicated comparisons. AU, arbitrary units a

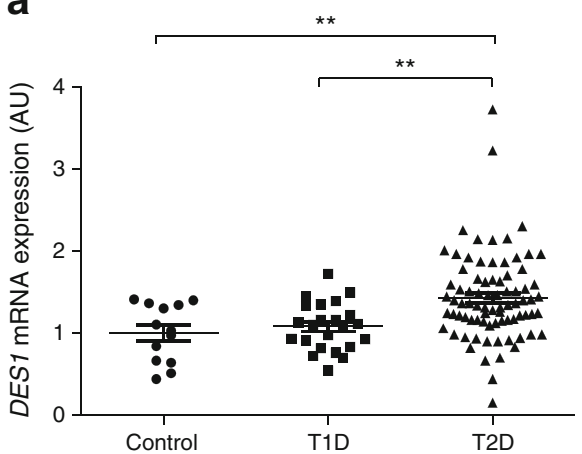

C

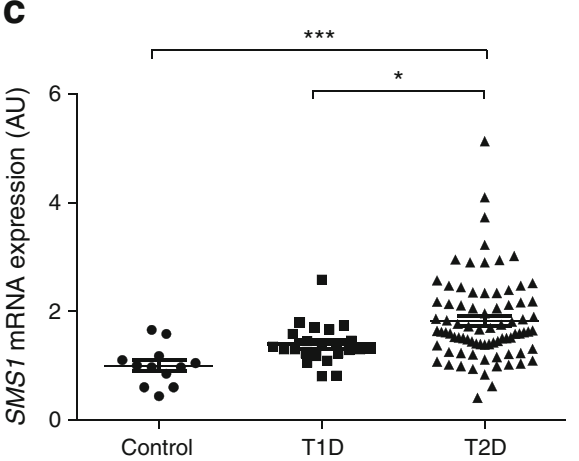

b

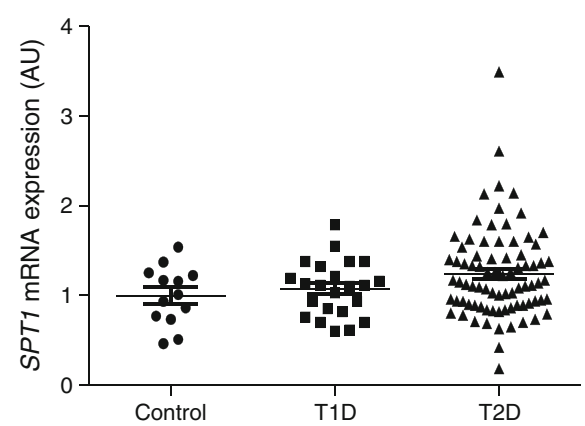

d

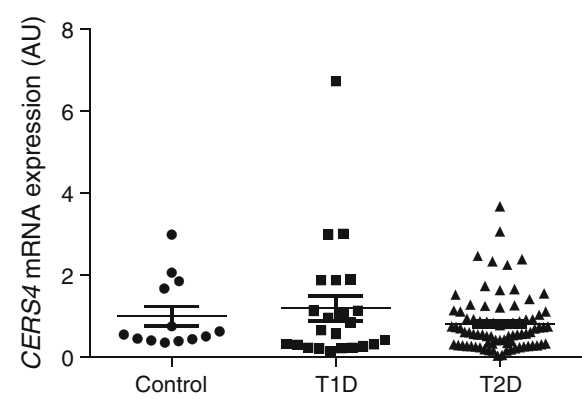

We show here that the markers of ER stress and TXNIP are increased in PBMCs of individuals with type 2 diabetes, an effect that cannot be solely related to hyperglycaemia since it is not detected in PBMCs of individuals with type 1 diabetes despite a higher blood glucose and $\mathrm{HbA}_{1 c}$. Moreover, TXNIP is not induced by a high glucose concentration in PBMCs of individuals with type 1 or type 2 diabetes. A concomitant increase in TXNIP and ER stress markers has already been described in PBMCs of individuals with type 2 diabetes [35]. We have several arguments to support the notion that this ER stress is causal in increasing the expression of TXNIP in human PBMCs, as already shown in pancreatic beta cells [6, 7], and in BMDMs of mice infected with Brucella abortus strain RB51 [36]. Decreasing the ER stress in PBMCs of individuals with type 2 diabetes by using a chemical chaperone reduces the expression of TXNIP. In a human macrophage cell line or in human BMDMs, ER stress inducers also induce TXNIP expression, an effect minimised in the presence of chemical chaperones.
Table 4 Clinical characteristics of the diabetic individuals involved in plasma lipid species determinations

\begin{tabular}{lll}
\hline Characteristic & Individuals with T1D $(n=35)$ & Individuals with T2D $(n=94)$ \\
\hline Age, years & $37 \pm 17$ & $58.7 \pm 11.0^{\dagger \dagger \dagger}$ \\
Sex, $n$ male/ $n$ female & $15 / 20$ & $43 / 18$ \\
BMI, $\mathrm{kg} / \mathrm{m}^{2}$ & $23.3 \pm 3$ & $30 \pm 5.4^{\dagger \dagger}$ \\
Diabetes duration, years & $14.7 \pm 14.0$ & $9.3 \pm 7.9^{\dagger}$ \\
Fasting plasma glucose, mmol/1 & $10.1 \pm 4.8$ & $8.5 \pm 2.6^{\dagger \dagger}$ \\
$\mathrm{HbA}_{1 \mathrm{c}}, \%$ & $8.5 \pm 2.3$ & $8.06 \pm 1.4$ \\
$\mathrm{HbA}_{1 \mathrm{c}}, \mathrm{mmol} / \mathrm{mol}$ & $69 \pm 18$ & $64 \pm 11$ \\
Triacylglycerols, mmol/1 & $0.94 \pm 0.42$ & $1.94 \pm 1.89^{\dagger \dagger \dagger}$ \\
Total cholesterol, mmol/1 & $47 \pm 10$ & $45 \pm 11$ \\
LDL-cholesterol, mmol/1 & $26 \pm 7$ & $25 \pm 11$ \\
HDL-cholesterol, mmol/1 & $16 \pm 5$ & $12 \pm 5$ \\
Total/HDL-cholesterol ratio & $2.9 \pm 0.7$ & $4.1 \pm 1.7^{\dagger \dagger \dagger}$ \\
\hline
\end{tabular}

The results are presented as mean \pm SD unless stated otherwise

${ }^{\dagger} p<0.05,{ }^{\dagger \dagger} p<0.01$ and ${ }^{\dagger \dagger} p<0.001$ vs T1D

T1D, type 1 diabetes; T2D, type 2 diabetes 
Fig. 6 Sphingolipid subspecies are increased in plasma of individuals with type 2 diabetes. Scatter plots show the concentration of sphingolipid subspecies from non-diabetic individuals (control, $n=13$ ), individuals with type 1 diabetes (T1D, $n=35$ ) and individuals with type 2 diabetes (T2D, $n=94$ ). Horizontal bars indicate the mean value \pm SEM. $* p<0.05$ and $* * * p<0.001$ for indicated comparisons

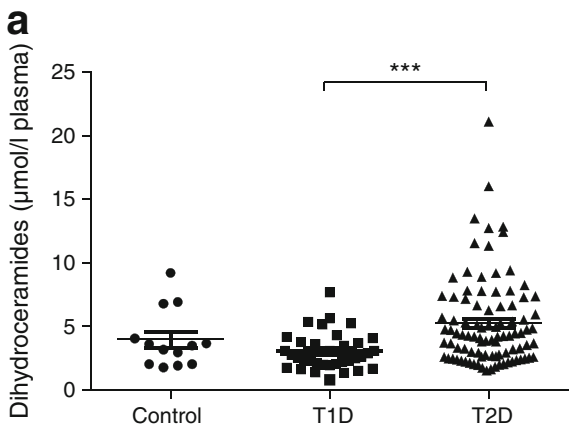

b
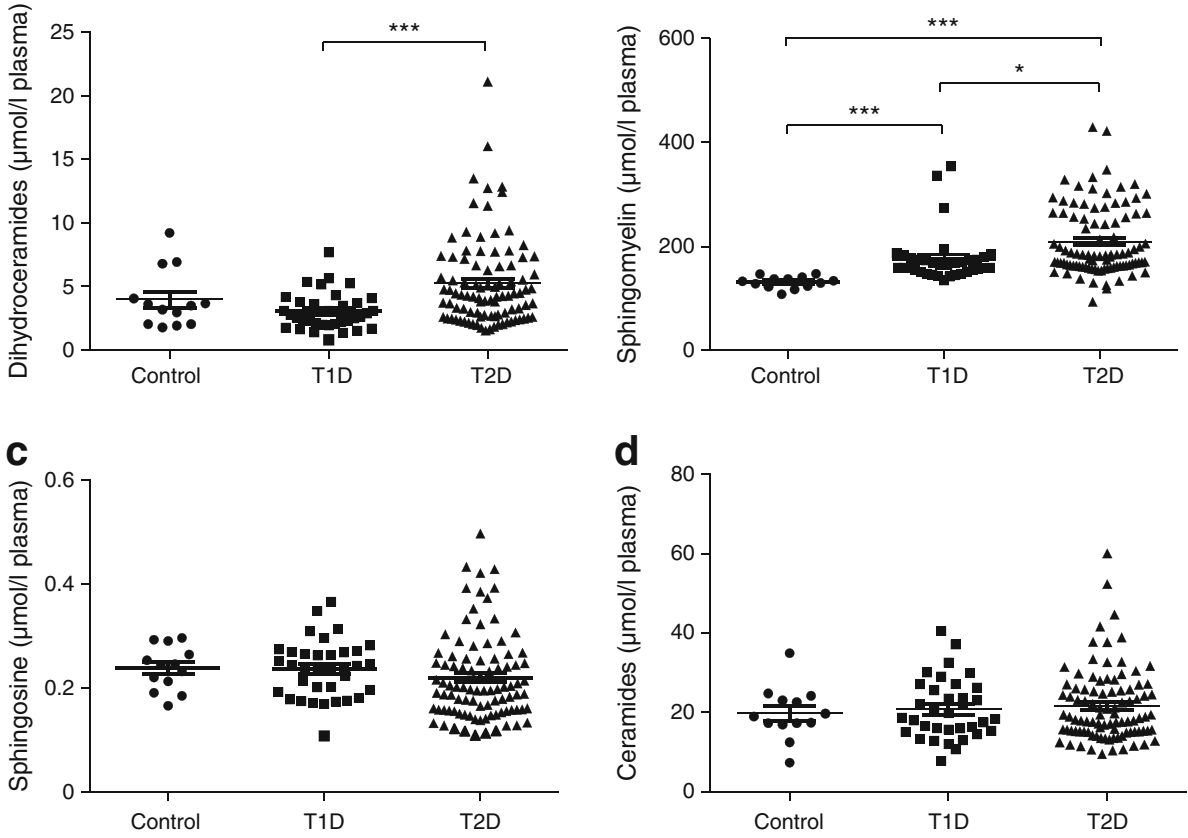

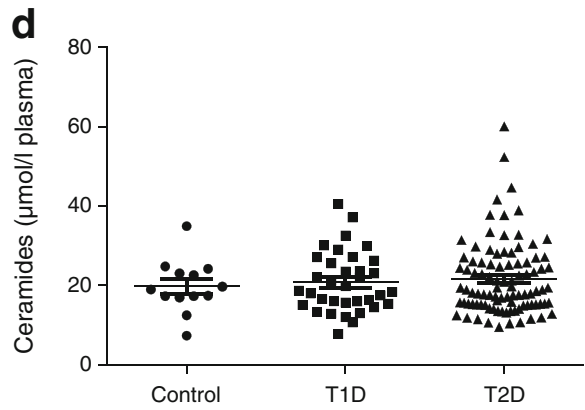

In the studies showing that ER stress induces TXNIP expression $[6,7,36]$, increased TXNIP mRNA results from transcriptional and/or post-transcriptional control. The PERK and/ or the IRE1 $\alpha$ branches of the UPR are involved in these effects whereas the ATF6 branch does not play a role. PERK activation leads to increased ChREBP expression, in turn inducing TXNIP transcription [7]. On the other hand, IRE1 $\alpha$ activation decreases the expression of miR-17, which induces TXNIP mRNA degradation [6]. In PBMCs of individuals with type 2 diabetes, the expression of the targets of the PERK branch and of the IRE1 $\alpha$ branch are increased but not the targets of the ATF6 branch, suggesting that the mechanisms described in pancreatic beta cells also hold true for immune cells.

In parallel with the increased expression of TXNIP, we find an increased expression of inflammatory markers, IL- $1 \beta$ and CCL2 in PBMCs of individuals with type 2 diabetes and a chemical chaperone that reduces ER stress and TXNIP expression is concomitant with reduced secretion of IL- $1 \beta$. Finally, decreasing the expression of TXNIP leads to reduced IL-1 $\beta$ secretion in macrophages.

The mechanism linking TXNIP and inflammasome activation implies a direct interaction between TXNIP and NLRP3 [8] and/or generation of reactive oxygen species by TXNIP linked to its inhibition of thioredoxin [6]. We find here that ER stress induces an increase in the protein content of NLRP3 in HMDMs. In PBMCs of individuals with type 2 diabetes there is also a significant increase in NLRP3 expression (Fig. 1b). Thus, ER stress could be involved, through TXNIP, in both inflammasome priming and activation. This dual effect of TXNIP has already been described in hepatocytes of rats fed a high-fructose diet [37].
Various lipid species including sphingolipids can induce ER stress. We show here that sphingolipid metabolism is probably altered in PBMCs of individuals with type 2 diabetes, since the expression of dihydroceramide desaturase and sphingomyelin synthase 1 (the most important sphingomyelin synthase isoform in macrophages [38]) is increased. The transcription of the latter enzyme is enhanced by saturated fatty acids such as palmitate [39]. An increase in the expression of enzymes involved in ceramide synthesis could be the consequence of activation of the Toll-like receptor (TLR) 4 by plasma saturated fatty acids [40] since in macrophages TLR4 activation induces de novo sphingolipid synthesis through activation of the expression of enzymes of the pathway [41].

Several studies have found a correlation between plasma ceramide concentrations, insulin resistance and inflammation [42]. One potential explanation is that ceramides act through TLR signalling [42]. Boon et al. [24] have shown that ceramide-enriched LDL can induce, in macrophages, a proinflammatory profile and that this is concomitant with an increased intracellular ceramide concentration.

Increased plasma concentrations of ceramide carried by lipoproteins have been found in small cohorts of obese individuals with type 2 diabetes [24], although not in all studies [43]. Here, in a larger cohort of overweight/obese individuals with type 2 diabetes, we found no differences in ceramides when this group was compared with nondiabetic individuals or individuals with type 1 diabetes but we did find increased saturated dihydroceramides species and decreased unsaturated species. Interestingly, similar plasma dihydroceramide species are associated with 


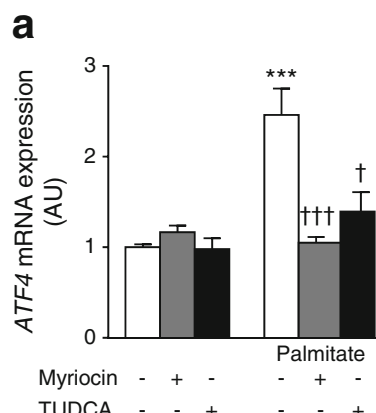

C

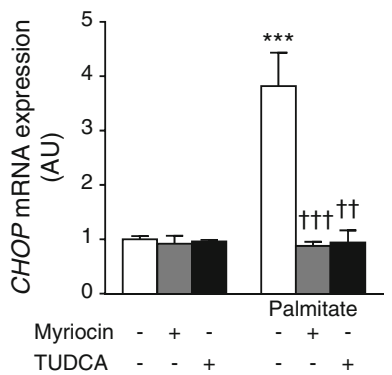

e

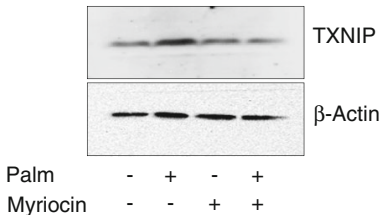

g

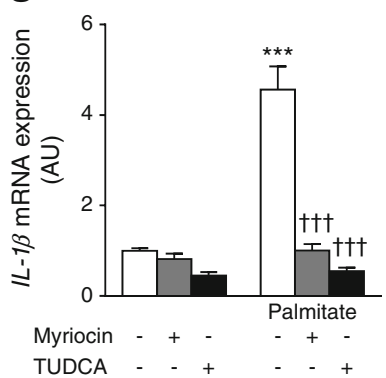

b

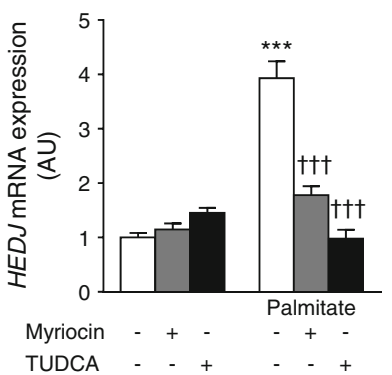

a

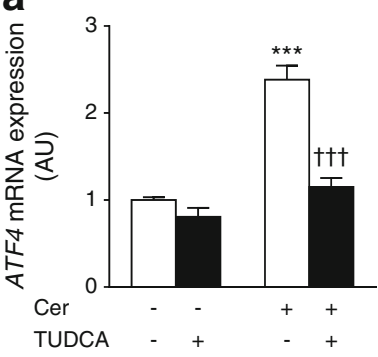

d

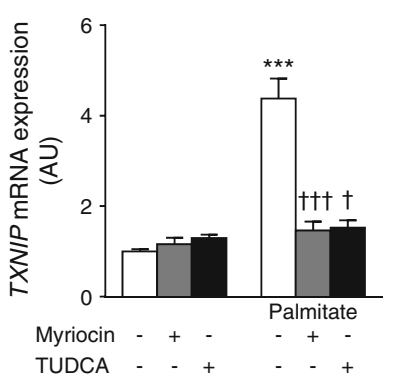

f

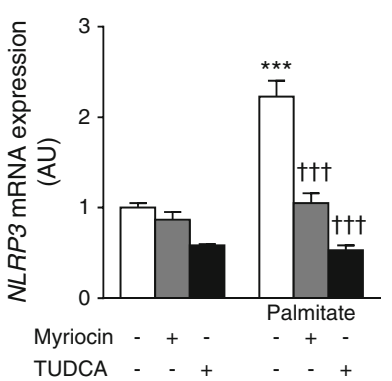

h
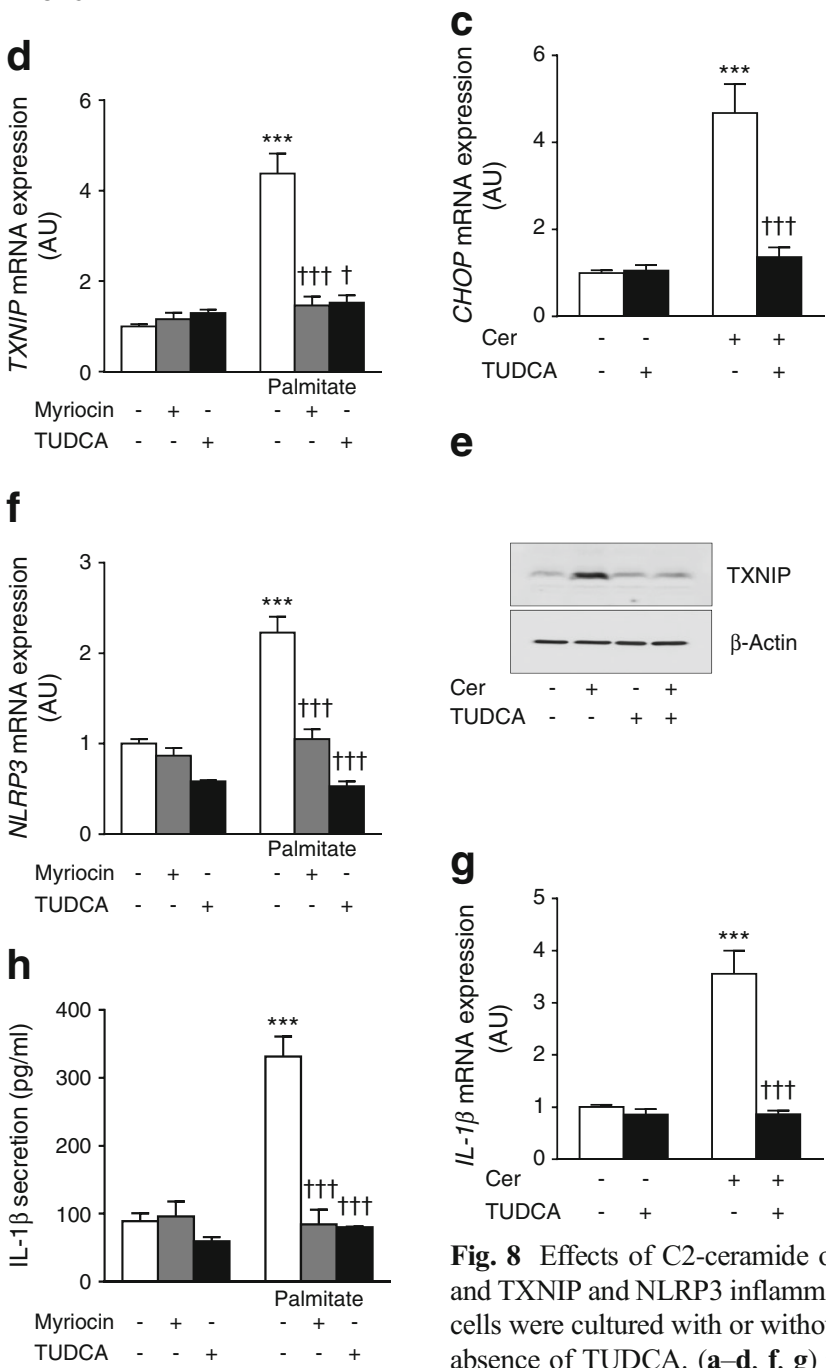

Fig. 7 Effects of palmitate on the expression of UPR markers and TXNIP and NLRP3 inflammasome activation in THP-1 cells. THP-1 cells were cultured with or without palmitate in the presence or absence of myriocin or TUDCA. (a-d, f, g) mRNA levels were quantified by qRTPCR. (e) Cell lysates were immunoblotted with antibodies against TXNIP and $\beta$-actin. (h) Medium from THP- 1 cells was used for the quantification of IL- $1 \beta$ secretion. Results are mean \pm SEM for three independent experiments; a representative blot is shown in (e). $* * * p<0.001$ vs untreated cells; ${ }^{\dagger} p<0.05,{ }^{\dagger} p<0.01$ and ${ }^{\dagger \dagger} p<0.001$ vs palmitate-treated cells. AU, arbitrary units; Palm, palmitate

waist circumference and with raised serum triacylglycerols and type 2 diabetes in Mexican American families [44, 45].
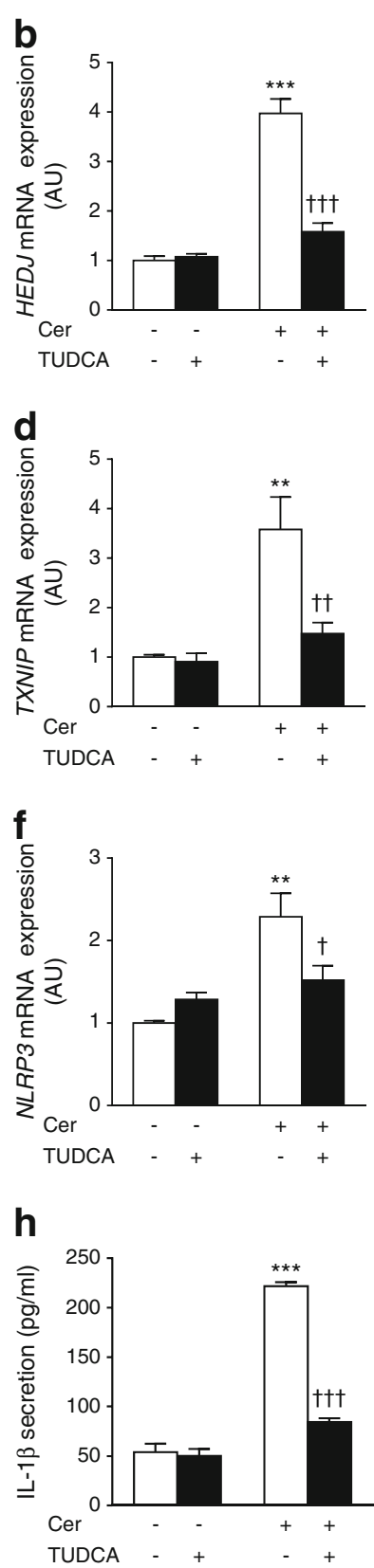

Fig. 8 Effects of C2-ceramide on the expression of ER stress markers and TXNIP and NLRP3 inflammasome activation in THP-1 cells. THP-1 cells were cultured with or without $\mathrm{C} 2$-ceramide (Cer) in the presence or absence of TUDCA. (a-d, f, $\mathbf{g})$ mRNA levels were quantified by qRTPCR. (e) Cell lysates were immunoblotted with antibodies against TXNIP and $\beta$-actin. (h) Medium from THP-1 cells was used for the quantification of IL-1 $\beta$ secretion. Results are mean \pm SEM for three independent experiments; a representative blot is shown in (e). $* * p<0.01$ and $* * * p<0.001$ vs untreated cells; ${ }^{\dagger} p<0.05,{ }^{\dagger \dagger} p<0.01$ and ${ }^{\dagger \dagger} p<0.001$ vs $\mathrm{C} 2$-ceramide condition. AU, arbitrary units

Thus, in addition to alterations in the expression of enzymes involved in endogenous sphingolipid metabolism, circulating immune cells of individuals with type 2 diabetes are exposed to a different plasma lipid environment. The two events could be related since exposure to specific lipids can 
modulate endogenous sphingolipid metabolism [24, 39]. In turn, endogenous lipids could be responsible for ER stress, enhanced TXNIP expression and inflammasome priming and activation as shown in in vitro experiments using macrophages.

In conclusion, we propose that quantitative and qualitative alterations in endogenous lipid metabolism and plasma lipid environment in individuals with type 2 diabetes orientate monocytes towards a proinflammatory state through an ER stress-TXNIP-inflammasome pathway.

Acknowledgements We are grateful to the donors for allowing us to use the blood samples and to the animal platform of the Centre de Recherche des Cordeliers for taking care of the animals. We thank $H$. Le Stunff (University Denis Diderot) for useful discussions, F. Fumeron (University Denis Diderot) for his help with statistical methods and F. Alzaïd (Centre de Recherche des Cordeliers) for editing the manuscript.

Data availability The data supporting the results reported in the article are available on request from the corresponding author.

Funding This work was supported by a grant from the 'Fondation pour la Recherche Médicale’ Equipe FRM DEQ20140329504.

Duality of interest $\mathrm{CB}$ is and $\mathrm{AK}$ was an employee of Servier Pharmaceutical. AS received a grant from Servier Pharmaceutical during the conduct of this study. All other authors declare that there is no duality of interest associated with their contribution to this manuscript.

Contribution statement AS contributed to the experimental design and data acquisition and wrote the manuscript. IH and AFB contributed to the experimental design and data acquisition and reviewed/edited the manuscript. NV contributed to the experimental design, interpretation of data and reviewed/edited the manuscript. AC, JFG and OB assisted in the collection of human specimens at Lariboisière and Pitié-Salpêtrière hospitals, interpretation of data and reviewed/edited the manuscript. OB also contributed to the conception and design of the study. EH contributed to interpretation of data and reviewed/edited the manuscript. $\mathrm{CB}$ and $\mathrm{AK}$ contributed to the experimental design and reviewed/edited the manuscript. PF contributed to the experimental design and data interpretation and wrote the manuscript. FF contributed to the experimental design and data analysis and interpretation, wrote the manuscript and is the guarantor of this work and, as such, had full access to all the data in the study. She takes responsibility for the integrity of the data and the accuracy of the data analysis. All authors approved the final version of the manuscript.

\section{References}

1. Osborn O, Olefsky JM (2012) The cellular and signaling networks linking the immune system and metabolism in disease. Nat Med 18: 363-374

2. Devevre EF, Renovato-Martins M, Clement K, Sautes-Fridman C, Cremer I, Poitou C (2015) Profiling of the three circulating monocyte subpopulations in human obesity. J Immunol 194:3917-3923

3. Donath MY, Shoelson SE (2011) Type 2 diabetes as an inflammatory disease. Nat Rev Immunol 11:98-107

4. Ehses JA, Perren A, Eppler E et al (2007) Increased number of isletassociated macrophages in type 2 diabetes. Diabetes 56:2356-2370
5. He Y, Hara H, Nunez G (2016) Mechanism and regulation of NLRP3 inflammasome activation. Trends Biochem Sci 41:10121021

6. Lerner AG, Upton JP, Praveen PVK et al (2012) IRE1 $\alpha$ Induces thioredoxin-interacting protein to activate the NLRP3 inflammasome and promote programmed cell death under irremediable ER stress. Cell Metab 16:250-264

7. Oslowski CM, Hara T, O Sullivan-Murphy B et al (2012) Thioredoxin-interacting protein mediates ER stress-induced $\beta$ cell death through initiation of the inflammasome. Cell Metab 16:265273

8. Zhou RB, Tardivel A, Thorens B, Choi I, Tschopp J (2010) Thioredoxin-interacting protein links oxidative stress to inflammasome activation. Nat Immunol 11:136-140

9. Nishiyama A, Matsui M, Iwata $S$ et al (1999) Identification of thioredoxin-binding protein-2/vitamin $\mathrm{D}_{3}$ up-regulated protein 1 as a negative regulator of thioredoxin function and expression. $\mathrm{J}$ Biol Chem 274:21645-21650

10. Shalev A (2014) Minireview: thioredoxin-interacting protein: regulation and function in the pancreatic $\beta$-cell. Mol Endocrinol 28: $1211-1220$

11. Kammoun HL, Chabanon H, Hainault I et al (2009) GRP78 expression inhibits insulin and ER stress-induced SREBP-1c activation and reduces hepatic steatosis in mice. J Clin Invest 119:1201-1215

12. Hage Hassan R, Hainault I, Vilquin JT et al (2012) Endoplasmic reticulum stress does not mediate palmitate-induced insulin resistance in mouse and human muscle cells. Diabetologia 55:204-214

13. Tran TTT, Postal BG, Demignot $\mathrm{S}$ et al (2016) Short term palmitate supply impairs intestinal insulin signaling via ceramide production. J Biol Chem 291:16328-16338

14. Parikh H, Carlsson E, Chutkow WA et al (2007) TXNIP regulates peripheral glucose metabolism in humans. PLoS Med 4:868-879

15. Shaked M, Ketzinel-Gilad M, Ariav Y, Cerasi E, Kaiser N, Leibowitz G (2009) Insulin counteracts glucotoxic effects by suppressing thioredoxin-interacting protein production in INS-1E beta cells and in Psammomys obesus pancreatic islets. Diabetologia 52: 636-644

16. Stoltzman CA, Peterson CW, Breen KT, Muoio DM, Billin AN, Ayer DE (2008) Glucose sensing by MondoA : Mlx complexes: a role for hexokinases and direct regulation of thioredoxin-interacting protein expression. Proc Natl Acad Sci U S A 105:6912-6917

17. Svoboda M, Tastenoy M, Zhang Y et al (2013) D-glucose- and 3-Omethyl-D-glucose-induced upregulation of selected genes in rat hepatocytes and INS1E cells: re-evaluation of the possible role of hexose phosphorylation. Mol Med Rep 8:829-836

18. Lee AH, Iwakoshi NN, Glimcher LH (2003) XBP-1 regulates a subset of endoplasmic reticulum resident chaperone genes in the unfolded protein response. Mol Cell Biol 23:7448-7459

19. Parker R, Phan T, Baumeister P et al (2001) Identification of TFII-I as the endoplasmic reticulum stress response element binding factor ERSF: its autoregulation by stress and interaction with ATF6. Mol Cell Biol 21:3220-3233

20. Yoshida H, Matsui T, Yamamoto A, Okada T, Mori K (2001) XBP1 mRNA is induced by ATF6 and spliced by IRE1 in response to ER stress to produce a highly active transcription factor. Cell 107:881891

21. Vandanmagsar B, Youm YH, Ravussin A et al (2011) The NLRP3 inflammasome instigates obesity-induced inflammation and insulin resistance. Nat Med 17:179-188

22. Hage Hassan R, Pacheco de Sousa AC, Mahfouz R et al (2016) Sustained action of ceramide on the insulin signaling pathway in muscle cells: implication of the double-stranded RNA-activated protein kinase. J Biol Chem 291:3019-3029

23. Senkal CE, Ponnusamy S, Manevich Y et al (2011) Alteration of ceramide synthase 6/C-16-ceramide induces activating transcription factor 6-mediated endoplasmic reticulum (ER) stress and 
apoptosis via perturbation of cellular $\mathrm{Ca}^{2+}$ and ER/Golgi membrane network. J Biol Chem 286:42446-42458

24. Boon J, Hoy AJ, Stark R et al (2013) Ceramides contained in LDL are elevated in type 2 diabetes and promote inflammation and skeletal muscle insulin resistance. Diabetes 62:401-410

25. Wellen KE, Hotamisligil GS (2005) Inflammation, stress, and diabetes. J Clin Invest 115:1111-1119

26. Laybutt DR, Preston AM, Akerfeldt MC et al (2007) Endoplasmic reticulum stress contributes to beta cell apoptosis in type 2 diabetes. Diabetologia 50:752-763

27. Cunha DA, Hekerman P, Ladriere L et al (2008) Initiation and execution of lipotoxic ER stress in pancreatic $\beta$-cells. J Cell Sci 121:2308-2318

28. Kharroubi I, Ladriere L, Cardozo AK, Dogusan Z, Cnop M, Eizirik DL (2004) Free fatty acids and Cytokines induce pancreatic betacell apoptosis by different mechanisms: role of nuclear factor-kappa $\mathrm{B}$ and endoplasmic reticulum stress. Endocrinology 145:50875096

29. Nakatani Y, Kaneto H, Kawamori D et al (2005) Involvement of endoplasmic reticulum stress in insulin resistance and diabetes. J Biol Chem 280:847-851

30. Ozcan U, Yilmaz E, Ozcan L et al (2006) Chemical chaperones reduce ER stress and restore glucose homeostasis in a mouse model of type 2 diabetes. Science 313:1137-1140

31. Hotamisligil GS (2010) Endoplasmic reticulum stress and the inflammatory basis of metabolic disease. Cell 140:900-917

32. Feng B, Yao PM, Li YK et al (2003) The endoplasmic reticulum is the site of cholesterol-induced cytotoxicity in macrophages. Nat Cell Biol 5:781-792

33. Erbay E, Babaev VR, Mayers JR et al (2009) Reducing endoplasmic reticulum stress through a macrophage lipid chaperone alleviates atherosclerosis. Nat Med 15:1383-1391

34. Robblee MM, Kim CC, Abate JP et al (2016) Saturated fatty acids engage an IRE1 $\alpha$-dependent pathway to activate the NLRP3 inflammasome in myeloid cells. Cell Rep 14:2611-2623

35. Lenin R, Sankaramoorthy A, Mohan V, Balasubramanyam M (2015) Altered immunometabolism at the interface of increased endoplasmic reticulum (ER) stress in patients with type 2 diabetes. J Leukoc Biol 98:615-622

36. Bronner DN, Abuaita BH, Chen XY et al (2015) Endoplasmic reticulum stress activates the inflammasome via NLRP3-and caspase-2-driven mitochondrial damage. Immunity 43:451-462

37. Zhang X, Zhang JH, Chen XY et al (2015) Reactive oxygen species-induced TXNIP drives fructose-mediated hepatic inflammation and lipid accumulation through NLRP3 inflammasome activation. Antioxid Redox Signal 22:848-870

38. Li ZQ, Fan YF, Liu J et al (2012) Impact of sphingomyelin synthase 1 deficiency on sphingolipid metabolism and atherosclerosis in mice. Arterioscler Thromb Vasc Biol 32:1577-1584

39. Hu W, Ross J, Geng TY, Brice SE, Cowart LA (2011) Differential regulation of dihydroceramide desaturase by palmitate versus monounsaturated fatty acids: implications for insulin resistance. J Biol Chem 286:16596-16605

40. Rocha DM, Caldas AP, Oliveira LL, Bressan J, Hermsdorff HH (2016) Saturated fatty acids trigger TLR4-mediated inflammatory response. Atherosclerosis 244:211-215

41. Sims K, Haynes CA, Kelly S et al (2010) $\mathrm{Kdo}_{2}$-Lipid A, a TLR4specific agonist, induces de novo sphingolipid biosynthesis in RAW264.7 macrophages, which is essential for induction of autophagy. J Biol Chem 285:38568-38579

42. Chavez JA, Summers SA (2012) A ceramide-centric view of insulin resistance. Cell Metab 15:585-594

43. Serlie MJ, Meijer AJ, Groener JE, Ackermans MT, Aerts JF, Sauerwein HP (2007) Chronic treatment with pioglitazone does not protect obese patients with diabetes mellitus type II from free fatty acid-induced insulin resistance. J Clin Endocrinol Metab 92: 166-171

44. Bellis C, Kulkarni H, Mamtani M et al (2014) human plasma lipidome is pleiotropically associated with cardiovascular risk factors and death. Circ-Cardiovasc. Gene 7:854-863

45. Mamtani M, Meikle PJ, Kulkarni H et al (2014) Plasma dihydroceramide species associate with waist circumference in Mexican American families. Obesity 22:950-956 